\title{
Energy required for weight maintenance in traumatic brain injury rehabilitation patients $v$. predictor equations
}

\author{
K.-A. McLaughlin ${ }^{1}$ and A. Holdoway ${ }^{2}$ \\ ${ }^{1}$ Dietetic Department, Royal National Hospital Rheumatic Diseases, Bath BAI 1RL, UK and ${ }^{2}$ Dietetic Department. \\ Royal United Hospital, Bath BAl 3NG, UK
}

There is an abundance of research on energy and protein required in the acute stage of traumatic brain injury (TBI), however, little relating to the rehabilitation stage ${ }^{(1)}$. When applying values derived from recommendations for energy required for weight maintenance ${ }^{(2)}$ and protein ${ }^{(3)}$ among TBI patients in long-term rehabilitation, undesirable weight gain has been observed. The aim of this study in patients with TBI in long-term rehabilitation and dependent on enteral feeding was to compare the energy and protein required to maintain an acceptable weight and BMI with the energy and protein estimated using Schofield ${ }^{(2)}$ and Elia ${ }^{(3)}$ data.

In this retrospective analysis, data from detailed dietetic case notes were reviewed for subjects in the period 2007-2009. Subjects were included if they met the following criteria: post TBI, nil by mouth, gastrostomy tube fed, had a BMI $=/>20 \mathrm{~kg} / \mathrm{m}^{2}$, weight stable for 4 weeks. Subjects were numbered to ensure anonymity. Data collected for each subject were age, weight, height, BMI, energy calculated using the Schofield ${ }^{(2)}$ equation, actual energy provided/d for weight maintenance and protein provided/d in using commercially available enteral feeds. Purposive sampling of patient records was employed to include those whose enteral feeding regimens maintained body weight for 4 weeks and whose feeding regimens were unchanged during this period. A paired $t$-test comparing energy provided with energy calculated using the Schofield ${ }^{(2)}$ equation was used to test the significance of any difference. Ethical approval was not required for this retrospective analysis. Results are quoted as mean (SD).

Data were analysed for 5 males, 5 females, mean age 41 years (SD 18). The table below summarises the mean (SD) for energy provided for weight maintenance ( $\mathrm{kJ}$ and kcal/d and $\mathrm{kJ}$ and $\mathrm{kcal} / \mathrm{kg}$ body weight/d) and energy calculated using Schofield ${ }^{(2)}(\mathrm{kJ}$ and $\mathrm{kcal})$. The result from the paired $t$-test as described above was $P=0.0005$. Daily protein provided from the commercial enteral feeds used in practice was $0.8 \mathrm{~g}(0.2 \mathrm{~g})$ per kg body weight.

\begin{tabular}{lcc}
\hline & Mean kJ (SD) & Mean kcal (SD) \\
\hline Daily energy provided & $5845(1151)$ & $1397(275)$ \\
Schofield daily energy & $8523(1000)$ & $2037(239)$ \\
Daily energy provided & $79(25)$ & $19(6)$ \\
per kg body weight & & \\
\hline
\end{tabular}

Although this study is small and retrospective, the results highlight that energy calculated by Schofield was significantly greater $(P<0.05)$ than that required in practice to maintain an acceptable weight in patients in the rehabilitation phase following TBI. This was true for all subjects. Using commercially available feeds protein intakes were comparable to the range suggested by Elia ${ }^{(3)}$ of $0.86-1.25 \mathrm{~g} / \mathrm{kg}$ body weight/d. While this study used weight stability as opposed to direct or indirect calorimetry to determine energy requirements, it demonstrates the discrepancy between predicted energy and energy required in clinical practice.

In the absence of more specific energy predictions, this study demonstrates the importance of regular monitoring of these patients to ensure feeding is adjusted to closely match energy requirements and avoid undesirable weight gain. Further research is warranted and a larger sample size may provide more accurate data to give an energy predictor equation for post TBI patients in the rehabilitation phase.

1. McEvoy CT, Cran GW, Cooke SR et al. (2009) Clin Nutr 28, 526-532.

2. Schofield WN (1985) Hum Nutr Clin Nutr 39, Suppl. 1, 5-41.

3. Elia M (1990) Artificial nutritional support. Med Int 82, 3392-3396. 